\title{
Antimicrobial Resistance and Molecular Characterization of Staphylococcus aureus Strains Causing Skin and Soft Tissue Infections in Greek Adults
}

\author{
S Mastraftsi ${ }^{1,2}$, S Sarrou ${ }^{1,2}$, K Pantelidi ${ }^{1,2}$, A Skoulakis ${ }^{1,2}$, A Roussaki ${ }^{3}$ and E Petinaki ${ }^{1,2 *}$ \\ ${ }^{1}$ Department of Microbiology, University Hospital of Larissa, Larissa, Greece \\ ${ }^{2}$ Department of Microbiology, Medical School, University of Thessaly, Larissa, Greece \\ ${ }^{3}$ Department of Dermatology, Medical School, University of Thessaly, Larissa, Greece
}

*Corresponding Author: E Petinaki, Professor, Head of Department of Microbiology, Medical School, University of Thessaly, Biopolis, Larissa, Greece.

Received: December 21, 2017; Published: January 19, 2018

DOI: 10.31080/ASMI.2018.01.0015

\begin{abstract}
During 2016, a total of one hundred twenty three Staphylococcus aureus isolates were collected from adult-patients skin and soft tissue infections (SSTIs) in Thessaly, Central Greece. The isolates were tested for susceptibility to various antimicrobial agents and the presence of resistance and virulence determinants. Resistance rates to cefoxitin, fusidic acid, erythromycin, clindamycin, tetracycline, ofloxacin and gentamycin were $22 \%, 36.6 \%, 29.3 \%, 26.8 \%, 22 \%, 7.3 \%$ and 4.8\% respectively, whereas, all were susceptible to vancomycin, teicoplanin, linezolid, mupirocin, trimethoprim-sulfamethoxazole and daptomycin. The presence of erm(A), tet(M) and $\operatorname{aac}\left(6^{\prime}\right)-\operatorname{Ie}-\operatorname{aph}\left(2^{\prime \prime}\right)$ genes were found in erythromycin/clindamycin, tetracycline and gentamicin-resistant isolates, respectively. Molecular characterization of isolates revealed the presence of two clones among MRSA (ST80-IV and ST225-II), while, among MSSA twelve different STs (ST1, 15, 45, 72, 7, 22, 728, 34, 59, 10, 398, 1153) were identified. The Panton-Valentine Leukocidin gene was detected in 39 strains, 18 MRSA which belonged to ST80, and 21 MSSA, which belonged to ST72 and ST728. The Toxic Shock Syndrome Toxin gene was found in ST34 MSSA. The results highlight the importance of both MRSA and MSSA as etiological agents in SSTIs, emphasizing the need for a close surveillance.
\end{abstract}

Keywords: Antimicrobial Resistance; Greece; Molecular Typing; Skin and Soft Tissue Infection; Staphylococcus aureus

\section{Introduction}

In the last years, skin and soft tissue infections (SSTIs) became one of the major causes of outpatient care and are associated with morbidity and substantial cost. Staphylococcus aureus is the most common cause of SSTIs worldwide, while a considerable rate is associated with methicillin-resistant S. aureus (MRSA) [1]. Although a large number of expert opinions, guidelines and recommendations for the management of SSTIs have been published over the last decades the change of epidemiology of staphylococcal infections may lead sometimes to the failure of antibiotic therapy [2].

In Greece, antimicrobial agents such as clindamycin, erythromycin, fusidic acid, tetracycline and trimethoprim-sulfamethoxazole are commonly used for the empiric therapy of suspected $S$. aureus skin and soft tissue infections [3].

\section{Aim of the Study}

Aim of the present study was to determine the rate of resistance to various antimicrobial agents and the molecular characterization of $S$. aureus isolated from SSTIs of adults in Greece, in order to understand the current epidemiology of $S$. aureus and so, to establish a more targeted empirical therapy.

\section{Materials and Methods}

During one year period (2016), one hundred twenty three (123) non-duplicate $S$. aureus isolates, were collected from a total of 353 skin or soft tissue infected outpatients, aged from 18 to 70 years old, all admitted to the Department of Dermatology of the University Hospital of Larissa (UHL). The Department of Dermatology of UHL is the reference center for dermatological diseases in Thessaly, a rural area in Central Greece with 1.200.000 inhabitants.

Criteria for patients' inclusion were clinical signs (impetigo, inflamed epidermal cyst, furuncles/carbuncles, cellulitis, folliculitis or other infections) combined with symptoms of infection including increased white blood cell counts, fever, redness, swelling and exudate. Demographic data (name, age, gender, residence, occupation, travel, previous hospitalization) and clinical information (underlying disease, antibiotic therapy) of the patients were collected. The research protocol was approved by the Ethics Committee of the General University Hospital of Larissa (Permit Number: 368); while, a written informed consent was obtained from the patients.

The clinical samples for culturing were obtained from the patient's abscesses and skin lesions using sterile swabs and were immediately sent to the Microbiological Laboratory. Identification to species level was based on colony morphology, Gram stain, cata- 
lase, coagulase production and detection of nuc gene. Susceptibility to various antimicrobial agents (benzylpenicillin, oxacillin, cefoxitin, clindamycin, erythromycin, fosfomycin, fusidic acid, gentamicin, levofloxacin, linezolid, ofloxacin, nitrofurantoin, rifampicin, teicoplanin, tetracycline, tigecycline, tobramycin, trimethoprimsulfamethoxazole and vancomycin) was assessed by using the automated system VITEK-2 (bioMerieux, Marcy l' Etoile, France); interpretation was done according to CLSI criteria (except fusidic acid and fosfomycin, for which EUCAST breakpoints were used) [4]. Determination of MICs to cefoxitin, daptomycin, mupirocin, vancomycin and teicoplanin was performed by Etest (bioMerieux, Marcy l'Etoile, France) according to manufacturer guidelines. Additionally, antimicrobial resistance genes $\left[m e c A, \operatorname{mec} C, \operatorname{erm}(\mathrm{A}), \operatorname{erm}(\mathrm{B}), \operatorname{erm}(\mathrm{C}), \operatorname{msr}(\mathrm{A}), \operatorname{tet}(\mathrm{M}), \operatorname{tet}(\mathrm{O}), \operatorname{tet}(\mathrm{L}), \operatorname{tet}(\mathrm{K}), \operatorname{aac}\left(6^{\prime}\right)-\right.$ Ie-aph(2")] as well as the genes encoding Panton-Valentine Leukocidin (lukS/lukFPV), Toxic Shock Syndrome Toxin-1 (tsst) and enterotoxins (sea, seb, sec, sed, see, seg, sei, seq, sek) were detected by PCR [5,6]. Positive and negative control strains were included in each PCR run. SCCmec types were identified by multiplex PCR amplification of the SCCmec region, while; molecular typing of all isolates was performed according to Multi Locus Sequence Typing (MLST) as described elsewhere $[7,8]$. Differences between MRSA and MSSA regarding the antimicrobial resistance rate and PVL presence were evaluated using $\chi^{2}$ or Fisher's exact tests. $\mathrm{P} \leq 0.05$ was considered statistically significant.

\section{Results}

Regarding the type of skin lesions 57 isolates were recovered from furuncle or carbuncle (46\%), 24 from folliculitis (19.5\%), 18 from impetigo (14.6\%), 18 from inflamed epidermal cyst (14.6\%) and 6 from cellulitis (4.8\%). The patients were 78 women (63.4\%) and 45 men (36.6\%) patients, between 18 and 70 years (mean age: $39 \pm 5$ years). All patients did not have hospitalization during the past year or risk factors and comorbidities associated with recent infection and surgery so, infections were characterized as community-onset.

According to susceptibility results for oxacillin and cefoxitin, 22\% of S. aureus (27 isolates) were classified as MRSA. Molecular typing of these strains revealed that 18 (66.7\% of MRSA) belonged to ST80-SCCmec IV, while the remaining 9 (33.3\%) belonged to ST225-SCCmec II. On the other hand, the molecular typing of the 96 MSSA strains revealed twelve different STs: ST1, ST15, ST45, ST72, ST7, ST22, ST728, ST34, ST59, ST10, ST398 and ST1153. In more details, ST1, ST15, ST45 and ST72 were the most prevalent ones accounting for 12 strains each (12.5\% of MSSA), followed by ST7, ST22 and ST728 identified in 9 strains each (9.3\%). The remaining MSSA strains belonged to ST34 and ST59, each corresponding to 6 strains (6.25\%), while ST10, ST398 and ST1153 corresponded to 3 strains each (3.12\%) (See Table 1 ).

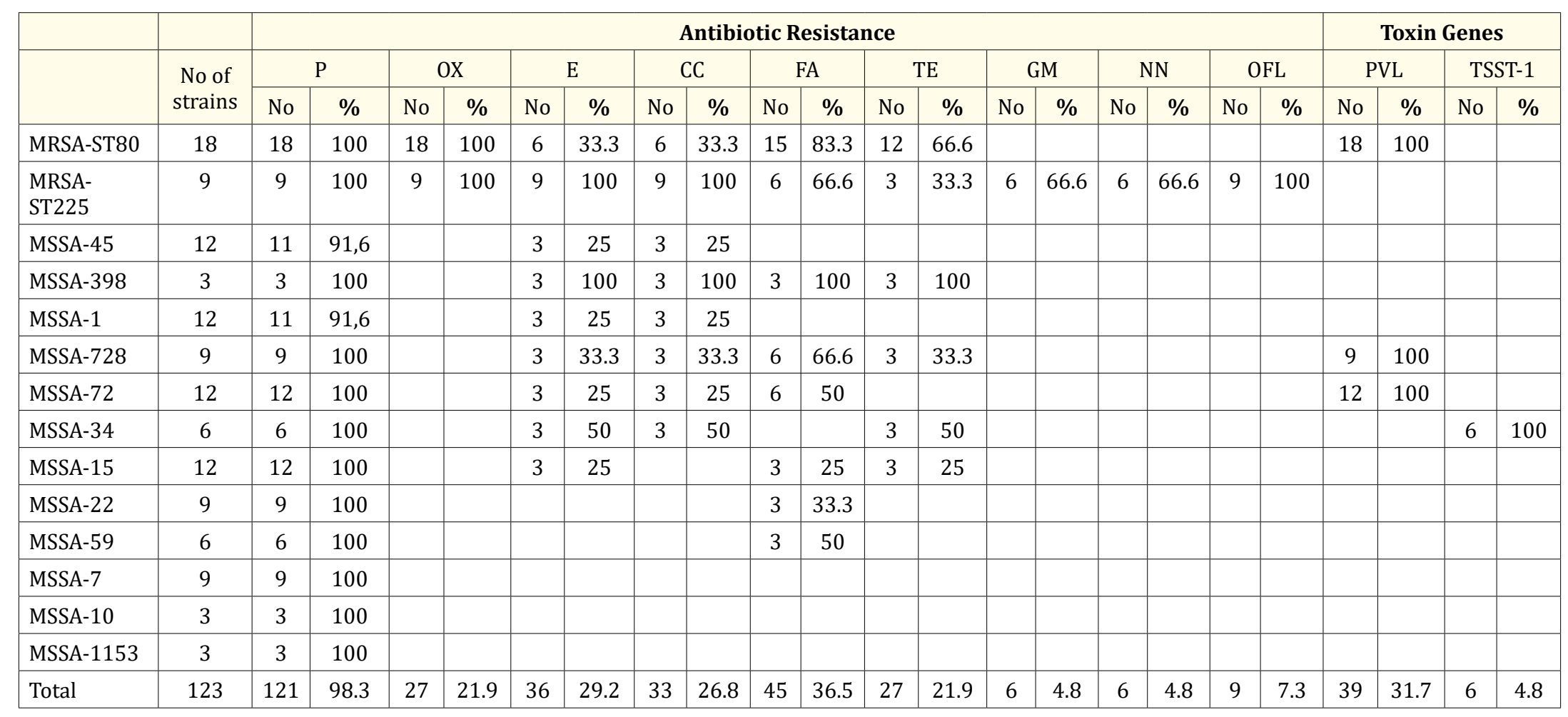

Table 1: Distribution of Staphylococcus aureus to different sequence types (STs), antimicrobial resistance rates and prevalence of PVL and TSST-1.

No: Number of strains; TE: Tetracycline; E: Erythromycin; CC: Clindamycin; FA: Fusidic Acid; OFX: Ofloxacin; OX: Oxacillin; P: Penicillin; GM: Gentamicin; NN: Tobramycin; PVL: Panton Valentine Leukocidin; TSST-1: Toxic Shock Syndrome Toxin-1

Both MRSA and MSSA of our collection were susceptible to vancomycin, teicoplanin, mupirocin, nitrofurantoin, rifampicin, linezolid, tigecycline, trimethoprimsulfamethoxazole, fosfomycin and daptomycin. The rate of resistance among MRSA and MSSA isolates is described on table 1. The resistance of MRSA strains seemed to be superior to erythromycin, clindamycin, tetracycline, fusidic acid, gentamycin, tobramycin and ofloxacin than MSSA (P $\leq 0.001)$. Resistance to cefoxitin, tetracycline and gentamycin/ tobramycin was associated with the presence of $\operatorname{mec} A, \operatorname{tet}(\mathrm{M})$ and aac(6')-Ie-aph (2") genes, respectively. In the group of the erythromycin-resistant strains, thirty three, which exhibited simultaneous resistance to erythromycin and clindamycin, were found to carry the $\operatorname{erm}(\mathrm{A})$ gene, while the remaining three, which were susceptible to clindamycin, carried the $m s r(\mathrm{~A})$ gene.

The PVL-gene was detected in 39 strains (31.7\%), while its presence was significant higher in MRSA than in MSSA (P $\leq 0.001)$. Among PVL-positive $S$. aureus, eighteen were MRSA and belonged to ST80, while, twenty-one were MSSA and belonged to ST72 and ST728 (twelve and nine respectively). Thirty-two (80\%) of 

Adults

the 39 PVL-positive strains were associated with furuncles or carbuncles, indicating the association of PVL with deep-seated SSTIs. Finally, the TSST-1 gene was found in all ST34 S. aureus. None of the remaining virulent genes was detected in any strain (See Table 1)

\section{Discussion}

In Greece, until now, the epidemiology of $S$. aureus isolated from SSTIs of adults is unknown, while the cost differs according to the chosen first line treatment [9]. Although previous national studies have shown the association of ST80 with skin infections, the present study demonstrates that multiple MSSA clones are also responsible $[3,7]$.

Recently, a survey from a pediatric Hospital in Greece was published underlining the presence of ST121 clone as responsible for the outbreak of SSTIs among children [10]. However, this clone was not detected in adults of our study. As confirmed in the present research, CA-MSSA skin infections can present with similar epidemiologic and clinical characteristics to CA-MRSA skin infections, a fact that has important clinical implications for the choice of empirical therapy of suspected community-acquired $S$. aureus skin and soft tissue infections.

PVL-positive $S$. aureus strains recovered from outpatients with SSTIs were also reported in Europe and worldwide [11-13]. However, differences are observed regarding firstly the percentage of PVL-positive strains (31.7\% in our study) and secondary the predominance of specific clones and susceptibility profile $[11,14]$. It is known that the epidemiology of $S$. aureus varies from continent to continent; the most prevalent genotypes are ST80-MRSA in Europe, ST30 in the southwest Pacific region, and ST8-MRSA in the USA and Japan $[11,14]$. So, it is very difficult to propose a common anti-staphylococcal therapy worldwide for the management of SSTIs [15].

In Greece, both MSSA and MRSA can play role as etiological agents of SSTIs. Close national surveillance of $S$. aureus strains by phenotypic and molecular identification procedures is essential to design a suitable treatment.

\section{Conclusion}

During 2016, a total of 123 Staphylococcus aureus isolates (27 MRSA and 96 MSSA) were collected from 353 adult-patients skin and soft tissue infections (SSTIs) in Thessaly, Central Greece (34.8\%). High rates of resistance to fusidic acid (36.5\%), erythromycin (29.2\%), clindamycin (26.8\%) and tetracycline (21.9\%) were detected. ST80 was the prevalent clone among MRSA, while, ST1, ST15, ST45 and ST72 were the most prevalent among MSSA. The PVL-gene was detected in 39 strains (31.7\%), while, its presence was significant higher in MRSA than in MSSA (P $\leq 0.001)$.

\section{Bibliography}

1. Esposito Silvano., et al. "Epidemiology and Microbiology of Skin and Soft Tissue Infections". Current Opinion in Infectious Diseases 29.2 (2016): 109-115.

2. Montravers Philippe., et al. "Current Guidelines and Recommendations for the Management of Skin and Soft Tissue Infections". Current Opinion in Infectious Diseases 29.2 (2016): 131-138.

3. Katopodis George D., et al. "Fusidic Acid and Clindamycin Resistance in Community-Associated, Methicillin-Resistant Staphylococcus Aureus Infections in Children of Central Greece". BMC Infectious Diseases 10 (2010): 351.

4. Clinical and Laboratory Standards Institute (CLSI). Performance Standards for Antimicrobial Susceptibility Testing: twenty six Informational Supplement M100-S26. CLSI, Wayne, PA: CLSI (2016).
5. Campbell Steven J., et al. "Genotypic Characteristics of Staphylococcus Aureus Isolates from a Multinational Trial of Complicated Skin and Skin Structure Infections". Journal of Clinical Microbiology 46.2 (2008): 678-684.

6. Liu Xiaoli., et al. "Molecular Characteristics of Community-Acquired Methicillin-Resistant Staphylococcus Aureus Strains Isolated from Outpatients with Skin and Soft Tissue Infections in Wuhan, China". Pathogens and Disease 74.4 (2016): ftw026

7. Drougka E., et al. "A 12-Year Survey of Methicillin-Resistant Staphylococcus aureus Infections in Greece: ST80-IV Epidemic?" Clinical Microbiology and Infection: The Official Publication of the European Society of Clinical Microbiology and Infectious Diseases 20.11 (2014): 0796-0803.

8. Enright MC., et al. "Multilocus Sequence Typing for Characterization of Methicillin-Resistant and MethicillinSusceptible Clones of Staphylococcus aureus". Journal of Clinical Microbiology 38.3 (2000): 1008-1015.

9. Athanasakis Kostas., et al. "Antibacterial Treatment of Meticillin-Resistant Staphylococcus Aureus Complicated Skin and Soft Tissue Infections: A Cost and Budget Impact Analysis in Greek Hospitals". Infectious Diseases and Therapy 3.2 (2014): 257-268.

10. Doudoulakakis Anastassios., et al. “Emergence of a Staphylococcus Aureus Clone Resistant to Mupirocin and $\mathrm{Fu}-$ sidic Acid Carrying Exotoxin Genes and Causing Mainly Skin Infections". Journal of Clinical Microbiology 55.8 (2017): 2529-2537.

11. Mine Yoshiko., et al. "Dissemination of Panton-Valentine Leukocidin-Positive Methicillin-Resistant Staphylococcus aureus in Okinawa, Japan". The Journal of Dermatology 40.1 (2013): 34-38

12. Yu Fangyou., et al. "Antimicrobial Susceptibility, Virulence Determinant Carriage and Molecular Characteristics of Staphylococcus Aureus Isolates Associated with Skin and Soft Tissue Infections". The Brazilian Journal of Infectious Diseases: An Official Publication of the Brazilian Society of Infectious Diseases 19.6 (2015): 614-622.

13. Takadama Shunsuke., et al. "Prevalence of Skin Infec tions Caused by Panton-Valentine Leukocidin-Positive Methicillin-Resistant Staphylococcus Aureus in Japan Particularly in Ishigaki, Okinawa". Journal of Infection and Chemotherapy: Official Journal of the Japan Society of Chemotherapy 23.11 (2017): 800-803.

14. Aung MS., et al. "Drug Resistance and Genetic Characteristics of Clinical Isolates of Staphylococci in Myanmar: High Prevalence of PVL among Methicillin-Susceptible Staphylococcus Aureus Belonging to Various Sequence Types". New Microbes and New Infections 10 (2016): 58-65.

15. VanEperen Alison S and John Segreti. "Empirical Therapy in Methicillin-Resistant Staphylococcus Aureus Infections: An Up-To-Date Approach". Journal of Infection and Chemotherapy: Official Journal of the Japan Society of Chemotherapy 22.6 (2016): 351-359.

\section{Volume 1 Issue 2 February 2018} (C) All rights are reserved by E Petinaki., et al. 